\title{
The role of postoperative radiotherapy in pediatric patients with grade II intracranial ependymomas: a population-based, propensity score-matched study
}

This article was published in the following Dove Press journal: Cancer Management and Research

\author{
Xiangyang Deng' \\ Dongdong Lin' \\ Lisheng Yu' \\ Xingxing $\mathrm{Xu}^{2}$ \\ Nu Zhang' \\ Hui Zhou' \\ Hansong Sheng' \\ Bo Yin' \\ Fengchun Lin' \\ Shangyu $\mathrm{Xu}^{\mathrm{I}}$ \\ Dandong $\mathrm{Li}^{\prime}$ \\ Junhao Fang' \\ Xiangqi Lu' \\ Jian Lin'
}

'Department of Neurosurgery, The Second Affiliated Hospital and Yuying Children's Hospital of Wenzhou Medical University, Wenzhou, China; ${ }^{2}$ Department of Physiology, School of Basic Medical Sciences, Wenzhou Medical University, Wenzhou, China

Correspondence: Jian Lin

Department of Neurosurgery, The

Second Affiliated Hospital and Yuying

Children's Hospital of Wenzhou Medical

University, 109 Xueyuan Road, Wenzhou,

Zhejiang, China

Tel +8657788002502

Email dxy623400630@163.com
Purpose: The main objectives of this study were to clarify the efficacy of postoperative radiotherapy (PORT) for pediatric intracranial grade II ependymomas (EPNs) and to explore whether various characteristics are associated with different outcomes in patients with and without PORT. Patients and methods: Data from patients younger than 18 years diagnosed with grade II intracranial EPNs and treated by surgery, with or without PORT, were obtained from the Surveillance, Epidemiology, and End Results (SEER) database (1973-2013 data set). Propensity score-matched analysis was conducted to balance clinical variables. Patient characteristics were stratified and analyzed.

Results: In total, data from 632 patients with grade II EPNs treated by cancer-directed surgery with or without PORT were obtained from the SEER database. Multivariable Cox analysis in the matched cohort suggested that undergoing PORT (overall survival [OS], $P=0.020$; cancerspecific survival [CSS], $P=0.031$ ), undergoing gross total resection (GTR; subtotal resection [STR] vs GTR; OS, $P<0.001$; CSS, $P<0.001$ ), and older age (OS, $P<0.001$; CSS, $P<0.001$ ) were the independent predictors of superior prognosis. Stratified analysis demonstrated that patient characteristics, including infratentorial location, younger age, and STR, were associated with benefit from PORT, while the survival advantage was not detected in patients who underwent GTR. Conclusion: Propensity score-matched analysis using SEER data indicates survival advantages of PORT. Given the strong prognostic associations with extent of resection and patient age, we recommend PORT for younger patients treated by STR.

Keywords: pediatric, oncology, SEER, radiotherapy

\section{Introduction}

Ependymomas (EPNs) are rare, neuroepithelial malignant brain tumors of the central nervous system (CNS), which account for $1.8 \%$ of all primary CNS tumors and up to $10 \%$ of brain malignancies in childhood. ${ }^{1}$ Moreover, EPNs are the third most prevalent brain tumors in childhood. ${ }^{2,3}$ Unlike EPNs in adults, which most commonly occur in the spine, EPNs are intracranial in $\sim 90 \%$ of pediatric patients, with about two-thirds of tumors arising within the posterior fossa. ${ }^{4}$ Histopathologically, WHO classification system categorizes EPNs into three groups: grade I (subependymoma and myxopapillary EPN), grade II, and grade III (anaplastic EPN). ${ }^{5}$ Among grade II EPNs, there are multiple histological variants, including cellular, tanycytic, clear cell, and papillary EPNs. ${ }^{5,6}$ The standard treatment for EPN varies by geographic location and treatment center; however, there is a consensus that surgery and radiotherapy (RT) 
are the cornerstone for the treatment of children. ${ }^{7,8}$ Postoperative radiotherapy (PORT) is reported to predict superior survival in anaplastic or infratentorial EPNs; ${ }^{9-11}$ however, there have been few detailed investigations of the contribution of PORT to survival in patients with intracranial grade II EPNs as a separate group. Moreover, those reports that are available are inconclusive, particularly regarding patients undergoing complete tumor resection. In addition, owing to the rarity of these tumors, most studies have covered mixed grade tumors, had small sample sizes, and had consequent limited statistical power.

The aims of this study were to clarify the impact of PORT on treatment of pediatric grade II EPNs and to explore whether various characteristics are associated with different outcomes with or without PORT. We used the Surveillance, Epidemiology, and End Results (SEER) database for this analysis, which is an authoritative nationwide cancer database in USA. The SEER database has limitations, and it is not possible to draw conclusions from the data due to selection bias and heterogeneity in the eligibility criteria; ${ }^{12}$ therefore, we used propensity score-matched (PSM) analysis, ${ }^{13}$ which is applied in oncology to assess treatment efficacies with the aim of minimizing selection bias, in our analysis on the impact of PORT. ${ }^{14}$

\section{Materials and methods Study population}

The SEER 18-Registry (1973-2013 data set) of the American National Cancer Institute was used in this study. Data were extracted from the database using SEER*Stat software (version 8.3.5). Site and histology codes from ICD for Oncology, Third Edition (ICD-O-3) were employed to identify cases. Patients younger than 18 years with a diagnosis of EPN, including cellular, clear cell, tanycytic, EPN not otherwise specified (NOS) (ICD-O-3 Code 9391), and papillary EPN (ICD-O-3 Code 9393), were identified in this study, as described previously. ${ }^{15}$ Primary tumor sites selected were classified as cerebrum (C71.0), frontal lobe (C71.1), temporal lobe (C71.2), parietal lobe (C71.3), occipital lobe (C71.4), ventricle, NOS (C71.5), cerebellum, NOS (C71.6), brain stem (C71.7), overlapping lesion of brain (C71.8), and brain, NOS (C71.9). All patients had been treated by cancer-directed surgery, with or without PORT, and EPN was the only or the first malignancy in these patients.

In the SEER database, trained coders used surgical procedure codes to determine the extent of resection. For the purpose of analysis, we recoded surgical procedure codes into four categories as previously described. ${ }^{15}$ Briefly, these categories included "biopsy" (pre-1998, code 02; 1998+, code 20); "subtotal resection (STR)" (pre-1998, codes 20, $30,35,40,55 ; 1998+$, codes 21,40$)$; “'gross total resection (GTR)" (pre-1998, codes 30, 50; 1998+, codes 30, 55), and “surgery, NOS" (pre-1998, code 90; 1998+, code 90). PORT was performed by all means, and the doses administered, targeting field, fraction, and modality were not recorded in the SEER database. Four tumor stage categories were included as follows: "localized", "regional", "distant", and "unknown". As described in the SEER database, localized neoplasms refer to a confined lesion; regional extension includes involvement of the surrounding meninges, bone (skull), blood vessels, nerves, multiple brain regions or hemispheres, and either supratentorial or infratentorial compared with their starting location. Distant extension included involvement of cerebrospinal fluid (CSF), nasal cavity, nasopharynx, posterior pharynx, or further contiguous extension. Other information, including sex, race, year of diagnosis, age, tumor site, and tumor histologic subtype, were also obtained. The SEER data are publicly accessible, and no additional approval from the institutional review board was required for this study.

\section{PSM analysis}

As patients were not stochastically included in the database, selection bias from baseline characteristics could affect analysis of the effects of PORT. Therefore, PSM was performed to balance clinical variables between the non-PORT and PORT groups. Propensity scores were calculated according to the range of each baseline covariate including sex, race, year of diagnosis, age, tumor site, tumor histologic subtype, tumor stage, and surgery type. Patients treated with PORT were matched with others, according to the values calculated using an algorithm determining the nearest neighbor and 1:1 matching without replacements. The caliper was set as 0.05 for the PSM analysis.

\section{Statistical analyses}

In this study, overall survival (OS) and cancer-specific survival (CSS) were the two primary endpoints of interest. Differences in the baseline characteristics of patients between the PORT and non-PORT groups were assessed using the chi-squared test or continuity correction, as appropriate. We generated Kaplan-Meier curves for descriptive visualization of survival, and the log-rank test was conducted to compare the unadjusted CSS and OS rates between the PORT and non-PORT groups. Univariate and multivariable Cox proportional hazard regression models were used to calculate HRs 
with 95\% CIs for CSS and OS. After subgroup analysis of surgery type, tumor site, tumor stage, and patient age, using multivariable Cox proportional hazard regression models, we combined the variables tumor site and surgery type to generate the subgroups, "supratentorial tumor+GTR" and "infratentorial tumor+GTR", for additional analysis. All statistical analyses were conducted using SPSS version 24.0 (IBM Corporation, Armonk, NY, USA). Forest plots summarizing the results of subgroup Cox analyses were drawn using GraphPad Prism version 7.0. $P$-values $\leq 0.05$ were considered statistically significant.

\section{Results}

\section{Baseline characteristics}

A total of 632 pediatric patients diagnosed with grade II EPN were identified in this study. Baseline patient, tumor, and treatment-related characteristics are presented in Table 1. The median age was 4 years (range, $0-17$ years), and 360 $(57.0 \%)$ patients were males. White patients accounted for the majority, comprising $80.5 \%$ of the entire population. Two hundred one (31.8\%) patients had tumors in the supratentorial region, while 248 (39.2\%) patients had tumors in the infratentorial region. Primary tumor stages, categorized as localized, regional, and distant, were observed in 466 (73.7\%), 122 (19.3\%), and 23 (3.6\%) patients, respectively. Concerning the type of surgery, 274 (43.4\%), 168 (26.6\%), and 69 (10.9\%) patients underwent GTR, STR, and biopsy, respectively. The PORT and non-PORT groups comprised $410(64.9 \%)$ and 222 (35.1\%) patients, respectively.

\section{Survival analysis of data before PSM}

The 5-year OS and CSS rates for the PORT vs non-PORT groups were $70.8 \%$ vs $62.1 \%$ and $72.8 \%$ vs $66.6 \%$, respectively, in the unmatched population. Kaplan-Meier curves of OS and CSS according to receipt of PORT are shown in Figure $1 \mathrm{~A}$ and $\mathrm{B}$. There was a significant difference between the PORT and non-PORT groups in OS (log-rank test; $P=0.043)$; however, there was no significant difference in CSS $(P=0.143)$. As illustrated in Figure 2, using the non-PORT group as the reference, univariate analysis of the unmatched cohort revealed that PORT was associated with longer OS (HR, 0.764; 95\% CI, 0.588-0.993; $P=0.044$ ); however, there was no association with CSS (HR, 0.812; 95\% CI, 0.614-1.075; $P=0.145)$. Analysis using a multivariate Cox proportional hazards model generated $\mathrm{HR}$ values for OS and CSS of 0.682 (95\% CI, 0.511-0.909; $P=0.009$ ) and 0.707 (95\% CI, 0.520-0.963; $P=0.028$ ), respectively, indicating a statistically significant association of PORT with superior
Table I Baseline characteristics of all patients

\begin{tabular}{|c|c|}
\hline Variables & n (\%) \\
\hline All patients & $632(100)$ \\
\hline \multicolumn{2}{|l|}{ Age (years) } \\
\hline Mean \pm SD & $5.99 \pm 0.20$ \\
\hline Median & 4 \\
\hline $0-5$ & $357(56.5)$ \\
\hline $6-11$ & $152(24.1)$ \\
\hline $12-17$ & $123(19.5)$ \\
\hline \multicolumn{2}{|l|}{ Gender } \\
\hline Male & $360(57.0)$ \\
\hline Female & $272(43.0)$ \\
\hline \multicolumn{2}{|l|}{ Race } \\
\hline White & $509(80.5)$ \\
\hline Black & $78(12.3)$ \\
\hline Others & $45(7.1)$ \\
\hline \multicolumn{2}{|l|}{ Year of diagnosis } \\
\hline $1973-1983$ & $82(13.0)$ \\
\hline $1984-1993$ & $122(19.3)$ \\
\hline 1994-2003 & $198(31.3)$ \\
\hline $2004-2013$ & $230(36.4)$ \\
\hline \multicolumn{2}{|l|}{ Histologic subtypes } \\
\hline Papillary ependymoma & $5(0.8)$ \\
\hline Ependymoma, NOS & $627(99.2)$ \\
\hline \multicolumn{2}{|l|}{ Tumor site } \\
\hline Supratentorial & $201(31.8)$ \\
\hline Infratentorial & $248(39.2)$ \\
\hline Others & $183(29.0)$ \\
\hline \multicolumn{2}{|l|}{ Stage } \\
\hline Localized & $466(73.7)$ \\
\hline Regional & $122(19.3)$ \\
\hline Distant & $23(3.6)$ \\
\hline Unknown & $21(3.3)$ \\
\hline \multicolumn{2}{|l|}{ Surgery type } \\
\hline GTR & $274(43.4)$ \\
\hline STR & $168(26.6)$ \\
\hline Biopsy & $69(10.9)$ \\
\hline Surgery, NOS & $121(19.1)$ \\
\hline \multicolumn{2}{|l|}{ Radiotherapy } \\
\hline PORT & $410(64.9)$ \\
\hline Non-PORT & $222(35.1)$ \\
\hline
\end{tabular}

Note: Mean values are presented as the mean \pm SD.

Abbreviations: GTR, gross total resection; NOS, not otherwise specified; PORT, postoperative radiotherapy; STR, subtotal resection.

survival. In addition, older age (OS, $P<0.001 ; \mathrm{CSS}, P<0.001)$ was an indicator of superior prognosis, whereas STR (OS, $P<0.001$; CSS, $P<0.001)$ was associated with worse survival (Table 2).

\section{Survival analysis of data after PSM}

Following PSM, there were 209 patients matched in each group, and no significant differences were observed between the PORT and non-PORT groups in characteristics used for matching (sex, race, year of diagnosis, age, tumor site, tumor histologic subtype, tumor stage, and surgery type; Table 3 ), 
indicating that potential selection bias associated with the receipt of PORT was minimized.

The 5-year OS and CSS rates for the PORT vs non-PORT groups were $70.7 \%$ vs $63.8 \%$ and $73.2 \%$ vs $68.2 \%$, respectively, in the matched population. Kaplan-Meier curves of unadjusted OS and CSS of the PORT vs non-PORT groups were also generated (Figure 3), and the log-rank test demonstrated no significant differences in survival rates in the two groups. Univariate analysis of OS (HR, 0.855; 95\% CI, 0.628-1.164; $P=0.319)$ and CSS (HR, 0.896; 95\% CI, $0.644-1.247 ; P=0.514)$ for the matched cohort also demonstrated no survival advantage associated with receiving

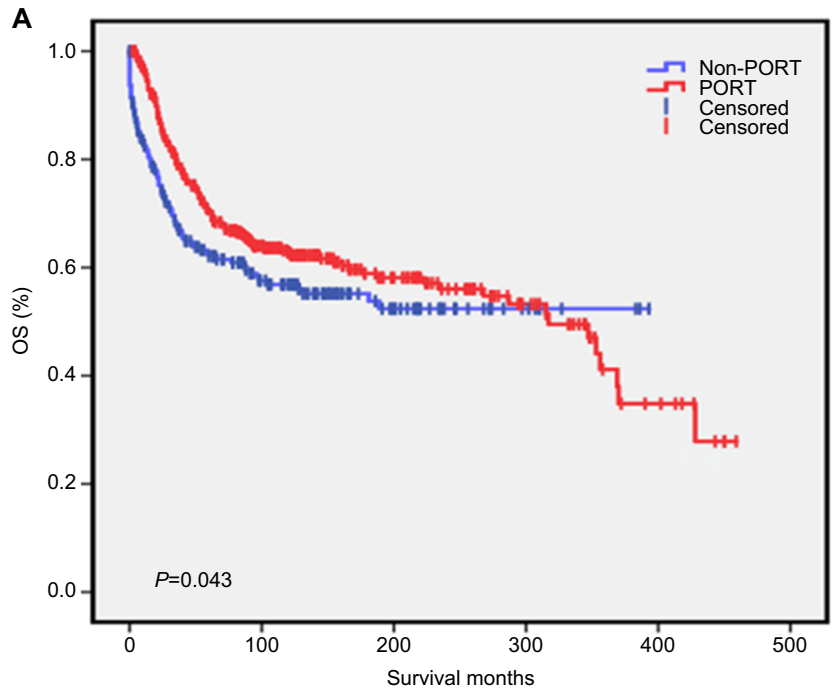

PORT; however, multivariable Cox analyses indicated that the HRs for PORT vs non-PORT relating to OS and CSS were 0.680 (95\% CI, 0.491-0.941;P=0.020) and 0.682 (95\% CI, 0.481-0.966; $P=0.031$ ), respectively, in favor of PORT (Figure 2). Older age (OS, $P<0.001$; CSS, $P<0.001$ ) was also an indicator of superior prognosis, whereas STR (OS, $P<0.001$; CSS, $P<0.001)$ was associated with inferior survival (Tables 4 ).

\section{Stratified subgroup analysis}

An exploratory subgroup analysis was conducted to identify the characteristics of patients who had benefited from



Figure I Kaplan-Meier survival curves illustrating OS (A) and CSS (B) of patients in the PORT and non-PORT groups before propensity score matching. Abbreviations: CSS, cancer-specific survival; OS, overall survival; PORT, postoperative radiotherapy.

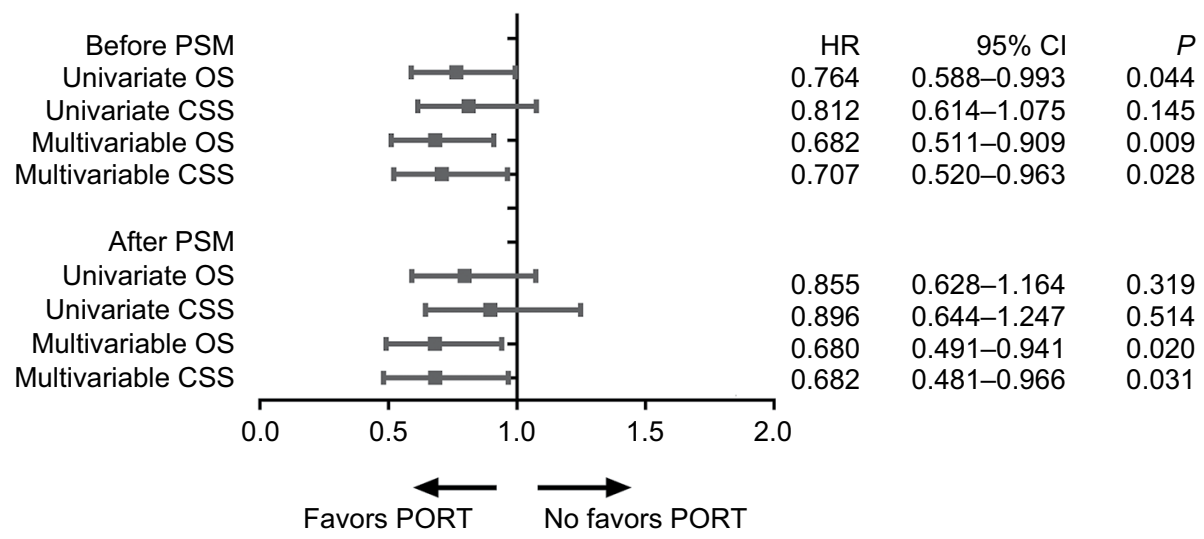

Figure 2 Forest plots illustrating the HR $(95 \% \mathrm{Cl})$ for OS and CSS calculated using the univariate and multivariable Cox proportional hazard regression models between patients in the PORT and non-PORT groups both before and after propensity score matching. The non-PORT group was used as the reference.

Abbreviations: CSS, cancer-specific survival; OS, overall survival; PORT, postoperative radiotherapy; PSM, propensity score matched. 

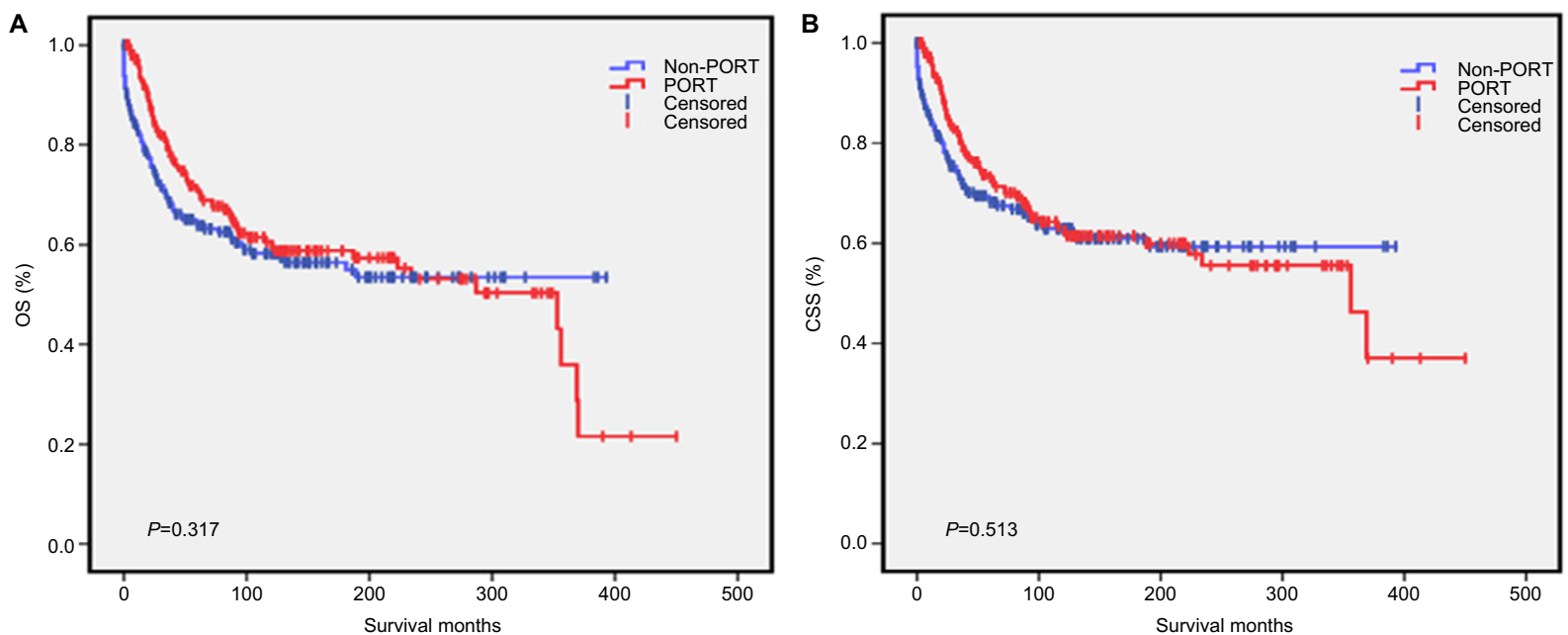

Figure 3 Kaplan-Meier survival curves illustrating OS (A) and CSS (B) of patients in the PORT and non-PORT groups after propensity score matching. Abbreviations: CSS, cancer-specific survival; OS, overall survival; PORT, postoperative radiotherapy.

Table 2 Multivariable Cox analyses before PSM

\begin{tabular}{|c|c|c|c|c|c|c|}
\hline \multirow[t]{2}{*}{ Variables } & \multicolumn{3}{|l|}{ OS } & \multicolumn{3}{|l|}{ CSS } \\
\hline & HR & $95 \% \mathrm{Cl}$ & $P$-value & HR & $95 \% \mathrm{Cl}$ & $P$-value \\
\hline \multicolumn{7}{|l|}{ Age (years) } \\
\hline $0-5$ & Ref & & & Ref & & \\
\hline $6-11$ & 0.740 & $0.530-1.033$ & 0.077 & 0.718 & $0.504-1.024$ & 0.067 \\
\hline $12-17$ & 0.411 & $0.263-0.642$ & $<0.001$ & 0.395 & $0.245-0.637$ & $<0.001$ \\
\hline \multicolumn{7}{|l|}{ Gender } \\
\hline Male & Ref & & & Ref & & \\
\hline Female & 0.913 & $0.70 \mathrm{I}-\mathrm{I} .188$ & 0.497 & 0.917 & $0.693-1.214$ & 0.546 \\
\hline \multicolumn{7}{|l|}{ Race } \\
\hline White & Ref & & & Ref & & \\
\hline Black & 1.054 & $0.706-1.574$ & 0.795 & 1.057 & $0.688-1.626$ & 0.800 \\
\hline Others & 0.827 & $0.484-1.413$ & 0.486 & 0.849 & $0.486-1.483$ & 0.566 \\
\hline \multicolumn{7}{|l|}{ Year of diagnosis } \\
\hline $1973-1983$ & Ref & & & Ref & & \\
\hline 1984-1993 & 0.591 & $0.366-0.953$ & 0.031 & 0.655 & $0.40 \mathrm{I}-\mathrm{I} .068$ & 0.090 \\
\hline 1994-2003 & 0.553 & $0.295-1.038$ & 0.065 & 0.713 & $0.369-1.378$ & 0.315 \\
\hline $2004-2013$ & 0.353 & $0.180-0.692$ & 0.002 & 0.448 & $0.221-0.909$ & 0.026 \\
\hline \multicolumn{7}{|l|}{ Histologic subtypes } \\
\hline Papillary ependymoma & Ref & & & Ref & & \\
\hline Ependymoma, NOS & 1.226 & $0.159-9.445$ & 0.845 & 1.230 & $0.158-9.584$ & 0.844 \\
\hline \multicolumn{7}{|l|}{ Tumor site } \\
\hline Supratentorial & Ref & & & Ref & & \\
\hline Infratentorial & 1.154 & $0.838-1.589$ & 0.381 & 1.156 & $0.825-1.620$ & 0.400 \\
\hline Others & 0.883 & $0.626-1.245$ & 0.477 & 0.832 & $0.576-1.201$ & 0.326 \\
\hline \multicolumn{7}{|l|}{ Stage } \\
\hline Localized & Ref & & & Ref & & \\
\hline Regional & 1.159 & $0.839-I .60 I$ & 0.371 & 1.154 & $0.816-1.632$ & 0.419 \\
\hline Distant & 1.534 & $0.794-2.962$ & 0.203 & 1.795 & $0.924-3.486$ & 0.084 \\
\hline Unknown & 1.914 & $1.021-3.589$ & 0.043 & 2.106 & $1.117-3.970$ & 0.021 \\
\hline \multicolumn{7}{|l|}{ Surgery type } \\
\hline GTR & Ref & & & Ref & & \\
\hline STR & 2.087 & $1.429-3.046$ & $<0.001$ & 2.079 & $1.390-3.109$ & $<0.001$ \\
\hline Biopsy & 1.069 & $0.584-1.960$ & 0.828 & 1.161 & $0.628-2.146$ & 0.633 \\
\hline Surgery, NOS & 2.446 & $1.347-4.443$ & 0.003 & 3.084 & I.649-5.769 & $<0.001$ \\
\hline \multicolumn{7}{|l|}{ Treatment } \\
\hline Non-PORT & Ref & & & Ref & & \\
\hline PORT & 0.682 & $0.5 I I-0.909$ & 0.009 & 0.707 & $0.520-0.963$ & 0.028 \\
\hline
\end{tabular}

Note: Bold values indicate statistical significance.

Abbreviations: CSS, cancer-specific survival; GTR, gross total resection; NOS, not otherwise specified; OS, overall survival; PORT, postoperative radiotherapy; PSM, propensity score matched; Ref, reference; STR, subtotal resection. 
Table 3 Characteristics of all patients before and after PSM

\begin{tabular}{|c|c|c|c|c|c|c|}
\hline \multirow[t]{2}{*}{ Variables } & \multicolumn{3}{|l|}{ Before PSM } & \multicolumn{3}{|l|}{ After PSM } \\
\hline & Non-PORT & PORT & $P$-value & Non-PORT & PORT & $P$-value \\
\hline All patients & $222(100)$ & $410(100)$ & & $209(100)$ & $209(100)$ & \\
\hline Age (years) & & & $<0.001$ & & & 0.597 \\
\hline Mean \pm SD & $4.77 \pm 0.34$ & $6.64 \pm 0.24$ & & $4.89 \pm 0.35$ & $5.99 \pm 0.33$ & \\
\hline Median & 2 & 5 & & 2 & 4 & \\
\hline $0-5$ & $150(67.6)$ & $207(50.5)$ & & $139(66.5)$ & $134(64.1)$ & \\
\hline $6-11$ & $35(15.8)$ & $117(28.5)$ & & \begin{tabular}{|l|}
$34(16.3)$ \\
\end{tabular} & $31(14.8)$ & \\
\hline $12-17$ & $37(16.7)$ & $86(21.0)$ & & $36(17.2)$ & $44(21 . I)$ & \\
\hline Gender & & & 0.155 & & & 0.433 \\
\hline Male & $\mathrm{II} 8(53.2)$ & $242(59.0)$ & & $113(54.1)$ & $105(50.2)$ & \\
\hline Female & $104(46.8)$ & $168(41.0)$ & & $96(45.9)$ & $104(49.8)$ & \\
\hline Race & & & 0.703 & & & 0.989 \\
\hline White & $178(80.2)$ & $331(80.7)$ & & $170(8 \mathrm{I} .3)$ & $169(80.9)$ & \\
\hline Black & $30(13.5)$ & 48 (II.7) & & $25(12.0)$ & $26(12.4)$ & \\
\hline Others & $14(6.3)$ & $31(7.6)$ & & $14(6.7)$ & $14(6.7)$ & \\
\hline Year of diagnosis & & & 0.014 & & & 0.243 \\
\hline $1973-1983$ & $19(8.6)$ & $63(15.4)$ & & $19(9.1)$ & $29(13.9)$ & \\
\hline $1984-1993$ & $49(22.1)$ & $73(17.8)$ & & $46(22.0)$ & $44(21.1)$ & \\
\hline 1994-2003 & $81(36.5)$ & $117(28.5)$ & & $73(34.9)$ & $58(27.8)$ & \\
\hline 2004-2013 & $73(32.9)$ & $157(38.3)$ & & \begin{tabular}{|l|}
$71(34.0)$ \\
\end{tabular} & 78 (37.3) & \\
\hline Histologic subtypes & & & 0.101 & & & 1.000 \\
\hline Papillary ependymoma & $4(1.8)$ & $\mathrm{I}(0.2)$ & & $\mathrm{I}(0.5)$ & $\mathrm{I}(0.5)$ & \\
\hline Ependymoma, NOS & $218(98.2)$ & $409(99.8)$ & & $208(99.5)$ & $208(99.5)$ & \\
\hline Tumor site & & & 0.204 & & & 0.948 \\
\hline Supratentorial & $78(35.1)$ & $123(30.0)$ & & $74(35.4)$ & $76(36.4)$ & \\
\hline Infratentorial & $77(34.7)$ & $17 \mid(41.7)$ & & $73(34.9)$ & 74 (35.4) & \\
\hline Others & $67(30.2)$ & $116(28.3)$ & & $62(29.7)$ & $59(28.2)$ & \\
\hline Stage & & & 0.322 & & & \begin{tabular}{|l|l|}
0.891 \\
\end{tabular} \\
\hline Localized & $156(70.3)$ & $310(75.6)$ & & $154(73.7)$ & $152(72.7)$ & \\
\hline Regional & $50(22.5)$ & $72(17.6)$ & & $44(21.1)$ & $43(20.6)$ & \\
\hline Distant & $10(4.5)$ & $13(3.2)$ & & $6(2.9)$ & $9(4.3)$ & \\
\hline Unknown & $6(2.7)$ & $15(3.7)$ & & $5(2.4)$ & $5(2.4)$ & \\
\hline Surgery type & & & 0.001 & & & 0.248 \\
\hline GTR & $104(46.8)$ & $170(4 I .5)$ & & $100(47.8)$ & $83(39.7)$ & \\
\hline STR & $73(32.9)$ & $95(23.2)$ & & $66(31.6)$ & $69(33.0)$ & \\
\hline Biopsy & $17(7.7)$ & $52(12.7)$ & & $15(7.2)$ & $16(7.7)$ & \\
\hline Surgery, NOS & $28(12.6)$ & $93(22.7)$ & & $28(13.4)$ & $4 I(19.6)$ & \\
\hline
\end{tabular}

Notes: Bold values indicate statistical significance. Mean values are presented as the mean \pm SD.

Abbreviations: GTR, gross total resection; NOS, not otherwise specified; PORT, postoperative radiotherapy; PSM, propensity score matched; STR, subtotal resection.

PORT (Figure 4). Patients who were younger than 5 years (OS: HR, 0.617; 95\% CI, 0.424-0.897; $P=0.012$; CSS: HR, $0.639 ; 95 \% \mathrm{CI}, 0.428-0.953 ; P=0.028)$ and whose tumors were located in the infratentorial region (OS: HR, 0.498; 95\% CI, 0.291-0.854; $P=0.011$; CSS: HR, 0.515; 95\% CI, $0.295-0.901 ; P=0.020)$ derived significantly more benefit from PORT treatment. Moreover, patients treated by STR may also benefit from PORT, as the calculated HR was 0.621 (95\% CI, 0.368-1.050; $P=0.075$ ) for OS, indicating borderline significance. The HR for CSS was 0.625 (95\% CI, 0.352-1.108; $P=0.108)$; however, the lack of statistical significance may be attributable to the small sample size.
There was also no significant influence of tumor stage on PORT effects on OS or CSS (all $P>0.05$ ). Moreover, neither the "supratentorial+GTR" group (OS: $P=0.474$; CSS: $P=0.941$ ) nor the "infratentorial+ GTR" group (OS: $P=0.442$; CSS: $P=0.442$ ) was significantly associated with differences in survival after PORT.

\section{Discussion}

To evaluate the potential for selection bias in analysis of the effects of PORT, both pre-PSM and post-PSM data were analyzed in our study. Post-PSM cohorts were matched according to patient, tumor, and treatment variables. We found that the 
Table 4 Multivariable Cox analyses after PSM

\begin{tabular}{|c|c|c|c|c|c|c|}
\hline \multirow[t]{2}{*}{ Variables } & \multicolumn{3}{|l|}{ OS } & \multicolumn{3}{|l|}{ CSS } \\
\hline & HR & $95 \% \mathrm{Cl}$ & $P$-value & HR & $95 \% \mathrm{Cl}$ & $P$-value \\
\hline \multicolumn{7}{|l|}{ Age (years) } \\
\hline $0-5$ & Ref & & & Ref & & \\
\hline $6-11$ & 0.630 & $0.377-1.054$ & 0.079 & 0.62 & $0.359-1.070$ & 0.086 \\
\hline $12-17$ & 0.359 & $0.208-0.618$ & $<0.001$ & 0.323 & $0.177-0.588$ & $<0.001$ \\
\hline \multicolumn{7}{|l|}{ Gender } \\
\hline Male & Ref & & & Ref & & \\
\hline Female & 0.800 & $0.577-1.110$ & 0.182 & 0.801 & $0.564-1.139$ & 0.217 \\
\hline \multicolumn{7}{|l|}{ Race } \\
\hline White & Ref & & & Ref & & \\
\hline Black & 0.628 & $0.362-1.088$ & 0.097 & 0.594 & $0.322-1.095$ & 0.095 \\
\hline Others & 1.346 & $0.7 \mid 4-2.539$ & 0.359 & 1.386 & $0.709-2.710$ & 0.339 \\
\hline \multicolumn{7}{|l|}{ Year of diagnosis } \\
\hline $1973-1983$ & Ref & & & Ref & & \\
\hline 1984-1993 & 0.530 & $0.279-1.005$ & 0.052 & 0.549 & $0.286-1.056$ & 0.072 \\
\hline 1994-2003 & 0.469 & $0.214-1.026$ & 0.058 & 0.582 & $0.258-1.313$ & 0.192 \\
\hline $2004-2013$ & 0.292 & $0.125-0.683$ & 0.004 & 0.362 & $0.149-0.877$ & 0.025 \\
\hline \multicolumn{7}{|l|}{ Histologic subtypes } \\
\hline Papillary ependymoma & Ref & & & Ref & & \\
\hline Ependymoma, NOS & 4.506 & $0.59 I-34.35 I$ & 0.146 & 5.410 & $0.700-41.780$ & 0.106 \\
\hline \multicolumn{7}{|l|}{ Tumor site } \\
\hline Supratentorial & Ref & & & Ref & & \\
\hline Infratentorial & 1.333 & $0.911-1.951$ & 0.139 & 1.357 & $0.907-2.029$ & 0.137 \\
\hline Others & 0.825 & $0.539-1.263$ & 0.376 & 0.739 & $0.465-1.176$ & 0.203 \\
\hline \multicolumn{7}{|l|}{ Stage } \\
\hline Localized & Ref & & & Ref & & \\
\hline Regional & 1.132 & $0.768-1.670$ & 0.531 & 1.161 & $0.764-1.765$ & 0.483 \\
\hline Distant & 1.155 & $0.498-2.680$ & 0.738 & 1.355 & $0.579-3.170$ & 0.483 \\
\hline Unknown & $\mathrm{I} .547$ & $0.559-4.280$ & 0.401 & 1.654 & $0.590-4.636$ & 0.338 \\
\hline \multicolumn{7}{|l|}{ Surgery type } \\
\hline GTR & Ref & & & Ref & & \\
\hline STR & 2.453 & I.57I-3.829 & $<0.001$ & 2.548 & $1.579-4.111$ & $<0.001$ \\
\hline Biopsy & 0.845 & $0.298-2.394$ & 0.751 & 0.973 & $0.34 \mathrm{I}-2.78 \mathrm{I}$ & 0.960 \\
\hline Surgery, NOS & 2.958 & $1.415-6.184$ & 0.004 & 4.027 & $1.861-8.718$ & $<0.001$ \\
\hline \multicolumn{7}{|l|}{ Treatment } \\
\hline Non-PORT & Ref & & & Ref & & \\
\hline PORT & 0.680 & $0.49 I-0.94 I$ & 0.020 & 0.682 & $0.48 \mathrm{I}-0.966$ & 0.031 \\
\hline
\end{tabular}

Note: Bold values indicate statistical significance.

Abbreviations: CSS, cancer-specific survival; GTR, gross total resection; NOS, not otherwise specified; OS, overall survival; PORT, postoperative radiotherapy; PSM, propensity score matched; Ref, reference; STR, subtotal resection.

PORT group had superior OS and CSS. By using multivariable Cox models, we found that administration of PORT was associated with superior survival in both the pre- and postPSM data sets, indicating favorable outcomes in response to RT in pediatric patients with grade II intracranial EPNs. This is consistent with previous reports, the majority of which have also found that PORT confers a survival advantage; however, study populations have included mixtures of patients with WHO grade II and III EPNs. ${ }^{9,16-20}$ In addition, response rates to photon beam RT in patients with grade II intracranial EPNs are reported to be greater than $80 \%$, emphasizing the efficacy of RT. ${ }^{21}$ In contrast, Aizer et a ${ }^{11}$ demonstrated that RT was not an independent prognostic factor for patients with grade II EPNs (RT vs non-RT; HR, 2.77; 95\% CI, 0.57-13.52); however, this SEER-based study included only 112 patients, the majority of whom were adults. Adult patients are inclined to be resistant to RT, relative to children, which may have masked the effects of RT in the children in their analysis. In addition to PORT, our analysis also found that the extent of resection and patient age had prognostic value. These findings are consistent with numerous previous studies, which have confirmed the survival advantage of gross resection of the 


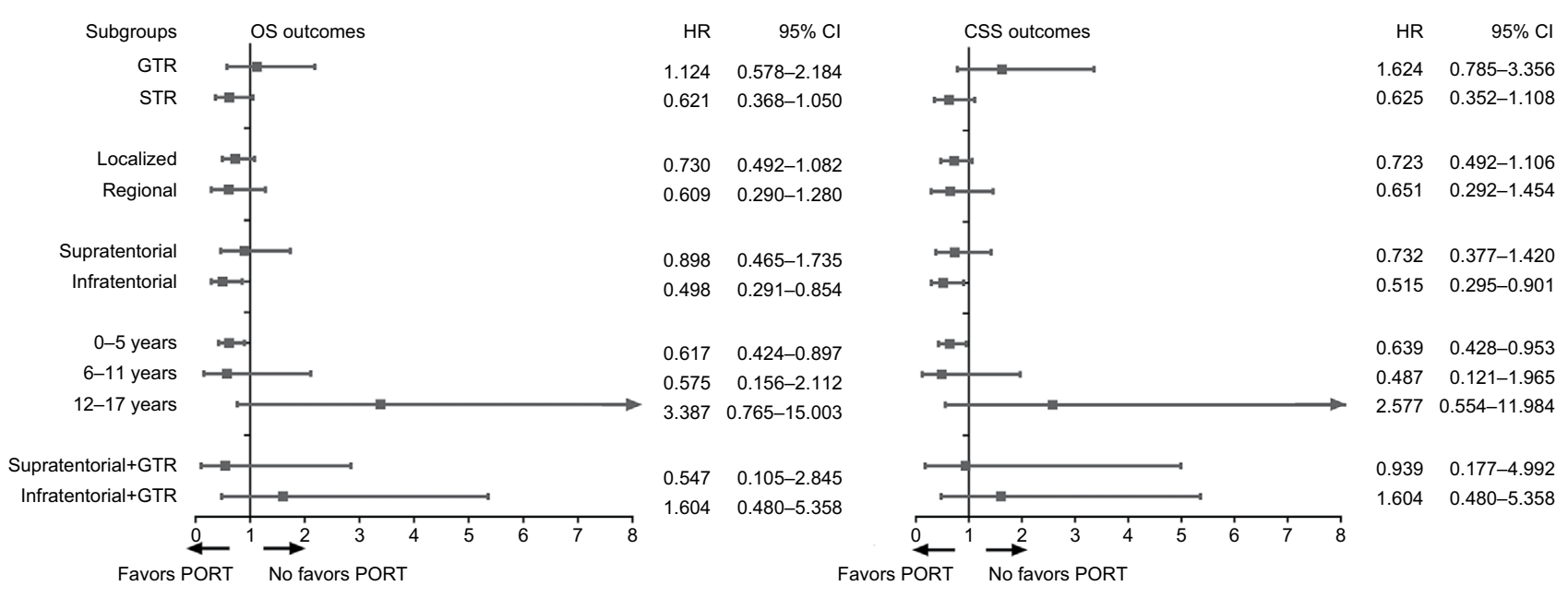

Figure 4 Forest plots illustrating HR $(95 \% \mathrm{Cl})$ for OS and CSS of each subgroup after propensity score matching. "Biopsy" and "distant" groups are not shown. Abbreviations: CSS, cancer-specific survival; GTR, gross total resection; OS, overall survival; PORT, postoperative radiotherapy; STR, subtotal resection.

total primary tumor and older age of children; $;^{4,7,8,20,22,23}$ however, tumor site and stage were not identified as independent prognostic factors in our study.

We also identified specific patient subgroups in which favorable effects of PORT were observed. GTR plus PORT had no apparent association with superior outcomes relative to GTR alone. Previous reports have suggested that GTR alone may confer superior survival outcomes for patients with grade II EPNs, ${ }^{11,24-26}$ whereas others found that PORT following GTR improved OS; ;,22,27 however, some of these studies also enrolled patients with anaplastic EPN, which is confirmed as requiring PORT, who made up the majority of participants in their studies. Recent studies suggested that adult patients with WHO grade II EPNs treated by GTR may also not require immediate RT if tumor spreading was not detected in the $\mathrm{CSF}^{28}$ One possible explanation is that EPNs tend to have discrete, pushing borders, rather than infiltrative borders, suggesting that some patients treated by GTR may not require adjuvant therapy. ${ }^{23}$ Moreover, patients who underwent STR were more likely to receive RT in our study, although the difference did not reach significance, probably because of the sample size. Given the importance of local tumor control, a consensus has been reached that incomplete surgery requires reinforcement by administration of additional radiation treatment.

We also found that tumors in the infratentorial region tended to be associated with superior outcomes in patients treated with PORT, indicating that patients with such tumors may benefit from radiation treatment, while there were no similar findings for tumors in the supratentorial region. Some studies have also shown that the lack of radiation treatment was associated with high recurrence rates and decreased survival in patients with infratentorial EPNs. ${ }^{10,11}$ This may be because gross total surgery is commonly more difficult for infratentorial lesions, due to involvement of the lower cranial nerves, brainstem, and vasculature, which often prevents complete removal of tumors. ${ }^{7,29}$ Among molecular genetic markers, gain of chromosome 1q is associated with EPNs in the posterior fossa, and this genomic aberration is also a significant predictor of tumor aggressiveness and poor prognosis. ${ }^{30} \mathrm{RT}$ has been suggested as an effective counteractive adjuvant therapy, despite the adverse effects of chromosome 1q25 gain; ${ }^{31}$ therefore, additional RT is necessary to prevent tumor recurrence and to improve patient outcomes. In further analysis, we evaluated the effects of PORT in patients with supratentorial tumors treated by GTR and found that it was not associated with survival. Supratentorial EPNs have different gene expression profiles compared with EPNs in other locations. The majority of nonsubependymal supratentorial EPNs carries YAP1 fusions and may have less aggressive characteristics, ${ }^{21}$ which could partially account for the lack of survival advantage of RT in supratentorial EPNs. Moreover, as RT can increase the risk of paralysis and other neurological side effects, our results suggest that it is reasonable to keep patients with grade II supratentorial EPNs under observation after GTR, rather than administering RT, consistent with previous reports. ${ }^{11}$ Our results also found no improvement in outcome in response to PORT for patients with infratentorial tumors treated by GTR; however, these findings should be considered with caution due to the relatively small sample size. Ailon et $\mathrm{al}^{26}$ reported that there was no evident inferior survival in 12 patients with WHO grade II posterior fossa 
EPN treated by GTR alone, compared with 57 treated by GTR plus PORT. Conversely, some authors have suggested that GTR is insufficient for the treatment of posterior fossa EPNs; ${ }^{10,11}$ however, optimal management protocols for EPNs in these situations have yet to be determined and require prospective evaluation.

It was also noted that younger pediatric patients were more likely to receive RT relative to older children in our study. Similar conclusions have also been drawn by other investigators. ${ }^{27}$ Paradoxically, younger children, especially those younger than 3 years, have the potential to experience significant long-term side effects from RT, attributable to their greater susceptibility to devastating neurocognitive, endocrinological, and neurological adverse effects. ${ }^{32-35}$ Therefore, it is crucial to minimize unnecessary exposure of young children, who require RT, to radiation. ${ }^{16}$ In recent years, proton therapy has been proposed as an alternative to conventional photon RT as it can reduce the total integral radiation dose and induce a significant decrease in the lowand intermediate-dose regions. ${ }^{36}$ Some studies have also reported that proton therapy provides a survival advantage for younger children. ${ }^{16,33,34}$ In addition, Grill et $\mathrm{al}^{37}$ and Grundy et $\mathrm{al}^{38}$ reported that a significant proportion of children younger than 3 years with EPNs could avoid or delay radiation treatment with adjuvant chemotherapy. Regardless, RT for children younger than 3 years remains a challenge and requires further research.

\section{Study strengths}

Our study has some strengths. First, the SEER data are unique resources and have been extensively validated..$^{39,40}$ The SEER database offers a large population-based cohort that was used for our study. Second, to our knowledge, this is the largest study evaluating the role of PORT in pediatric patients with grade II EPNs treated by surgery. Moreover, PSM was used to control for unmeasured factors, and any imbalance that might impact survival, improving the credibility of the results. OS and CSS were defined as the primary outcome measures and assessed by multivariable analysis both before and after propensity score matching. Stratified subgroup analysis was performed to identify independent factors associated with the efficacy of PORT.

\section{Study limitations}

There were also limitations to our analysis that should be acknowledged. First, the study was not a randomized controlled trial, and there were selection biases that could be controlled in part by study design and PSM, but not eliminated. In addition, the SEER registry data are retrospective and depend on the accuracy of local reporting; hence, any analysis of these data has limitations. The SEER database does not provide detailed information on RT parameters, such as dose, field, or duration. It also lacks information on adjuvant chemotherapy and has limited data on other factors. Moreover, information about surgery is recorded in the SEER database according to the clinical practice in USA at the time of its collection, which may differ significantly from current practice, which could also introduce bias. Finally, alterations in the histological classification criteria for grade II EPNs over time may have introduced heterogeneity.

\section{Conclusion}

Our propensity-matched analysis, based on the SEER database, indicates that PORT confers a survival advantage for patients with grade II intracranial EPNs. This study further highlights the importance of maximal resection for survival and verifies that patient age is an independent prognostic factor. Additional subgroup analysis suggests that patients with particular characteristics, including infratentorial tumor location, younger age, and non-total excision, would benefit from PORT; however, there was no survival advantage of PORT for patients who had undergone complete resection. Further investigations are required to refine the detailed treatment indications for PORT of grade II intracranial EPNs, particularly in children younger than 3 years.

\section{Acknowledgments}

The work was supported by Wenzhou Municipal Science and Technology Bureau (2014Y0105) and Research Start-up Project (89217022).

\section{Disclosure}

The authors report no conflicts of interest in this work.

\section{References}

1. Korshunov A, Witt H, Hielscher T, et al. Molecular staging of intracranial ependymoma in children and adults. J Clin Oncol. 2010;28(19):3182-3190.

2. Lillard JC, Venable GT, Khan NR, et al. Pediatric supratentorial ependymoma: surgical, clinical, and molecular analysis. Neurosurgery. Epub 2018 Jun 16

3. Mueller S, Chang S. Pediatric brain tumors: current treatment strategies and future therapeutic approaches. Neurotherapeutics. 2009;6(3):570-586.

4. Marinoff AE, Ma C, Guo D, et al. Rethinking childhood ependymoma: a retrospective, multi-center analysis reveals poor long-term overall survival. J Neurooncol. 2017;135(1):201-211.

5. Wen PY, Huse JT. World Health Organization classification of central nervous system tumors. Continuum (Minneap Minn). 2017;23(6):1531-1547.

6. Wu J, Armstrong TS, Gilbert MR. Biology and management of ependymomas. Neuro Oncol. 2016;18(7):902-913. 
7. Rudà R, Reifenberger G, Frappaz D, et al. EANO guidelines for the diagnosis and treatment of ependymal tumors. Neuro Oncol. 2018;20(4):445-456.

8. Merchant TE. Current clinical challenges in childhood ependymoma: a focused review. J Clin Oncol. 2017;35(21):2364-2369.

9. Merchant TE, Li C, Xiong X, Kun LE, Boop FA, Sanford RA. Conformal radiotherapy after surgery for paediatric ependymoma: a prospective study. Lancet Oncol. 2009;10(3):258-266.

10. Rogers L, Pueschel J, Spetzler R, et al. Is gross-total resection sufficient treatment for posterior fossa ependymomas? J Neurosurg. 2005;102(4):629-636.

11. Aizer AA, Ancukiewicz M, Nguyen PL, et al. Natural history and role of radiation in patients with supratentorial and infratentorial WHO grade II ependymomas: results from a population-based study. J Neurooncol. 2013;115(3):411-419.

12. Lim YJ, Song C, Kim JS. Improved survival with postoperative radiotherapy in thymic carcinoma: a propensity-matched analysis of Surveillance, Epidemiology, and End Results (SEER) database. Lung Cancer. 2017;108:161-167.

13. D'Agostino RB. Propensity score methods for bias reduction in the comparison of a treatment to a non-randomized control group. Stat Med. 1998;17(19):2265-2281.

14. Bendifallah S, Koskas M, Ballester M, Genin AS, Darai E, Rouzier R. The survival impact of systematic lymphadenectomy in endometrial cancer with the use of propensity score matching analysis. Am J Obstet Gynecol. 2012;206(6):500.e1-500.e11.

15. Lin Y, Jea A, Melkonian SC, Lam S. Treatment of pediatric Grade II spinal ependymomas: a population-based study. J Neurosurg Pediatr. 2015;15(3):243-249.

16. Macdonald SM, Sethi R, Lavally B, et al. Proton radiotherapy for pediatric central nervous system ependymoma: clinical outcomes for 70 patients. Neuro Oncol. 2013;15(11):1552-1559.

17. Merchant TE, Mulhern RK, Krasin MJ, et al. Preliminary results from a phase II trial of conformal radiation therapy and evaluation of radiationrelated CNS effects for pediatric patients with localized ependymoma. J Clin Oncol. 2004;22(15):3156-3162.

18. Rodríguez D, Cheung MC, Housri N, Quinones-Hinojosa A, Camphausen $\mathrm{K}$, Koniaris LG. Outcomes of malignant CNS ependymomas: an examination of 2408 cases through the Surveillance, Epidemiology, and End Results (SEER) database (1973-2005). J Surg Res. 2009;156(2):340-351.

19. Mansur DB, Perry A, Rajaram V. Postoperative radiation therapy for grade II and III intracranial ependymoma. Int J Radiat Oncol Biol Phys. 2005;61(2):387-391.

20. Shu HK, Sall WF, MaityA, et al. Childhood intracranial ependymoma: twentyyear experience from a single institution. Cancer. 2007;110(2):432-441.

21. Vitanza NA, Partap S. Pediatric ependymoma. J Child Neurol. 2016;31(12):1354-1366.

22. Snider CA, Yang K, Mack SC, et al. Impact of radiation therapy and extent of resection for ependymoma in young children: A populationbased study. Pediatr Blood Cancer. 2018;65(3):e26880.

23. Pejavar S, Polley MY, Rosenberg-Wohl S, et al. Pediatric intracranial ependymoma: the roles of surgery, radiation and chemotherapy. J Neurooncol. 2012;106(2):367-375.

24. Cage TA, Clark AJ, Aranda D, et al. A systematic review of treatment outcomes in pediatric patients with intracranial ependymomas. $J \mathrm{Neu}$ rosurg Pediatr. 2013;11(6):673-681.

Cancer Management and Research

Publish your work in this journal

Cancer Management and Research is an international, peer-reviewed open access journal focusing on cancer research and the optimal use of preventative and integrated treatment interventions to achieve improved outcomes, enhanced survival and quality of life for the cancer patient. The manuscript management system is completely online and includes
25. Hukin J, Epstein F, Lefton D, Allen J. Treatment of intracranial ependymoma by surgery alone. Pediatr Neurosurg. 1998;29(1):40-45.

26. Ailon T, Dunham C, Carret AS, et al. The role of resection alone in select children with intracranial ependymoma: the Canadian Pediatric Brain Tumour Consortium experience. Childs Nerv Syst. 2015;31(1): 57-65.

27. Koshy M, Rich S, Merchant TE, Mahmood U, Regine WF, Kwok Y. Post-operative radiation improves survival in children younger than 3 years with intracranial ependymoma. J Neurooncol. 2011;105(3): 583-590.

28. Metellus P, Guyotat J, Chinot O, et al. Adult intracranial WHO grade II ependymomas: long-term outcome and prognostic factor analysis in a series of 114 patients. Neuro Oncol. 2010;12(9):976-984.

29. Little AS, Sheean T, Manoharan R, Darbar A, Teo C. The management of completely resected childhood intracranial ependymoma: the argument for observation only. Childs Nerv Syst. 2009;25(3):281-284.

30. Yao Y, Mack SC, Taylor MD. Molecular genetics of ependymoma. Chin J Cancer. 2011;30(10):669-681.

31. Kilday JP, Mitra B, Domerg C, et al. Copy number gain of 1q25 predicts poor progression-free survival for pediatric intracranial ependymomas and enables patient risk stratification: a prospective European clinical trial cohort analysis on behalf of the Children's Cancer Leukaemia Group (CCLG), Societe Francaise d'Oncologie Pediatrique (SFOP), and International Society for Pediatric Oncology (SIOP). Clin Cancer Res. 2012;18(7):2001-2011.

32. Conklin HM, Li C, Xiong X, Ogg RJ, Merchant TE. Predicting change in academic abilities after conformal radiation therapy for localized ependymoma. J Clin Oncol. 2008;26(24):3965-3970.

33. Macdonald SM, Yock TI. Proton beam therapy following resection for childhood ependymoma. Childs Nerv Syst. 2010;26(3):285-291.

34. Indelicato DJ, Bradley JA, Rotondo RL, et al. Outcomes following proton therapy for pediatric ependymoma. Acta Oncol. 2018;57(5): 644-648.

35. Sato M, Gunther JR, Mahajan A, et al. Progression-free survival of children with localized ependymoma treated with intensity-modulated radiation therapy or proton-beam radiation therapy. Cancer. 2017;123(13):2570-2578.

36. Hall MD, Bradley JA, Rotondo RL, et al. Risk of radiation vasculopathy and stroke in pediatric patients treated with proton therapy for brain and skull base tumors. Int J Radiat Oncol Biol Phys. 2018;101(4):854-859.

37. Grill J, Le Deley MC, Gambarelli D, et al. Postoperative chemotherapy without irradiation for ependymoma in children under 5 years of age: a multicenter trial of the French Society of Pediatric Oncology. J Clin Oncol. 2001;19(5):1288-1296.

38. Grundy RG, Wilne SA, Weston CL, et al. Primary postoperative chemotherapy without radiotherapy for intracranial ependymoma in children: the UKCCSG/SIOP prospective study. Lancet Oncol. 2007;8(8):696-705.

39. Warren JL, Klabunde CN, Schrag D, Bach PB, Riley GF. Overview of the SEER-Medicare data: content, research applications, and generalizability to the United States elderly population. Med Care. 2002;40(8 Suppl):IV-3-18.

40. Bach PB, Guadagnoli E, Schrag D, Schussler N, Warren JL. Patient demographic and socioeconomic characteristics in the SEERMedicare database applications and limitations. Med Care. 2002;40(8 Suppl):IV-19-25.

a very quick and fair peer-review system, which is all easy to use. Visit http://www.dovepress.com/testimonials.php to read real quotes from published authors. 